COMMUNICATIONS IN

ANALYSIS AND GEOMETRY

Volume 12, Number 4, 927-947, 2004

\title{
The Apollonian Inner Metric
}

\author{
Peter A. HëstÖ
}

In this paper we derive an explicit formula for the inner metric of the Apollonian metric and prove that for most domains there exists a geodesic connecting two arbitrary points. We also give a necessary and sufficient condition for the Apollonian inner metric to be bilipschitz equivalent to the quasihyperbolic metric.

\section{Introduction.}

In this paper we consider the inner metric of the Apollonian metric from [3]. Some basic features of this metric are that it is Möbius invariant, equals the hyperbolic metric in balls and half-spaces and has a geodesic connecting an arbitrary pair of points (in most domains). We start by defining the Apollonian metric and its inner metric in order to state our main results. The notation used conforms largely to that of [2] and [19], the reader can consult Section 2.1 of this paper, if necessary.

We will be considering domains (open connected non-empty sets) $G$ in the Möbius space $\overline{\mathbb{R}^{n}}:=\mathbb{R}^{n} \cup\{\infty\}$. The Apollonian metric is defined for $x, y \in G \varsubsetneqq \mathbb{R}^{n}$ by

$$
\alpha_{G}(x, y):=\sup _{a, b \in \partial G} \log \frac{|a-x|}{|a-y|} \frac{|b-y|}{|b-x|}
$$

(with the understanding that $|\infty-x| /|\infty-y|=1$ ). It is in fact a metric if only if the complement of $G$ is not contained in a hyperplane and a pseudometric otherwise, as was noted in [3, Theorem 1.1]. This metric was introduced in [3] and has also been considered in [5, 8-14, 16, 17]. It should also be noted that the same metric has been considered from a different perspective under the name of the Barbilian metric for instance in [4] and [15].

\footnotetext{
${ }^{1} 2000$ Mathematics Subject Classification. Primary 30F45; Secondary 30C65.

${ }^{2}$ Supported in part by the Academy of Finland.
} 
Let $\gamma:[0,1] \rightarrow G \subset \mathbb{R}^{n}$ be a path, i.e. a continuous function. If $d$ is a metric in $G$, then the $d$-length of $\gamma$ is defined by

$$
d(\gamma):=\sup \sum_{i=0}^{k-1} d\left(\gamma\left(t_{i}\right), \gamma\left(t_{i+1}\right)\right)
$$

where the supremum is taken over $k<\infty$ and all sequences $\left\{t_{i}\right\}$ satisfying $0=t_{0}<t_{1}<\ldots<t_{k}=1$. All the paths in this paper are assumed to be rectifiable, that is have finite Euclidean length. The inner metric of $d$ is defined by the formula

$$
\tilde{d}(x, y):=\inf _{\gamma} d(\gamma)
$$

where the infimum is taken over all paths connecting $x$ and $y$ in $G$. We denote the inner metric of the Apollonian metric by $\tilde{\alpha}_{G}$ and call it the Apollonian inner metric.

Since the Apollonian metric is only a pseudometric in some domains it is not reasonable to expect the Apollonian inner metric to be a metric in every domain. The next theorem shows that it is a proper metric in a somewhat larger class of domains than is the Apollonian metric.

Theorem 1.2. The Apollonian inner metric is a pseudometric in every domain $G \varsubsetneqq \mathbb{R}^{n}$ and a metric if and only if the complement of $G$ is not contained in an $(n-2)$-dimensional plane.

In order to present an integral formula for the Apollonian inner metric we need the Apollonian spheres and the density of the Apollonian metric, both from [8].

Definition 1.3. Let $G \varsubsetneqq \mathbb{R}^{n}, x \in G$ and $\theta \in \mathbb{R}^{n} \backslash\{0\}$.

1. Let $r:=r(x, \theta):=\sup \left\{s: B^{n}(x+s \theta /|\theta|, s) \subset G\right\}$. We define the Apollonian sphere through $x$ in direction $\theta$ to be $S^{n-1}(x+r \theta /|\theta|, r)$ if $r<\infty$ and the limiting half-space otherwise.

2. We denote $\bar{\alpha}_{G}(x ; \theta):=1 /(2 r(x, \theta))+1 /(2 r(x,-\theta))$ and call this function the density of the Apollonian metric.

The main result of the present paper is the following integral formula for the Apollonian inner metric. Piecewise continuously differentiable means continuously differentiable except in a finite number of points. 
Theorem 1.4. If $x, y \in G \varsubsetneqq \mathbb{R}^{n}$, then

$$
\tilde{\alpha}_{G}(x, y)=\inf _{\gamma} \int \bar{\alpha}_{G}\left(\gamma(t) ; \gamma^{\prime}(t)\right)\left|\gamma^{\prime}(t)\right| d t,
$$

where the infimum is taken over all paths connecting $x$ and $y$ in $G$ that are piecewise continuously differentiable (with the understanding that $\bar{\alpha}_{G}(z ; 0) 0=0$ for all $z \in G$, even when $\bar{\alpha}_{G}(z ; 0)$ is not defined $)$.

The next result shows that the infimum in the definition of the Apollonian inner metric is a minimum in almost all cases, namely, when $\alpha_{G}$ is a metric.

Theorem 1.5. Let $x, y \in G \varsubsetneqq \mathbb{R}^{n}$ and $G$ be such that $G^{c}$ is not contained in a hyperplane. Then there exists a path $\gamma$ connecting $x$ and $y$ in $G$ such that $\tilde{\alpha}_{G}(\gamma)=\tilde{\alpha}_{G}(x, y)$.

In the last section of this paper we consider inequalities between the Apollonian inner metric, the $j_{G}$ metric and the quasihyperbolic metric, $k_{G}$. Let $G \varsubsetneqq \mathbb{R}^{n}$ be a domain and $x, y \in G$. The $j_{G}$ metric, which is a modification from [18] of a metric from [6], is defined by

$$
j_{G}(x, y):=\log \left\{1+\frac{|x-y|}{\min \{d(x, \partial G), d(y, \partial G))\}}\right\} .
$$

The quasihyperbolic metric from [7] is defined by

$$
k_{G}(x, y):=\inf _{\gamma} \int \frac{|d z|}{d(z, \partial G)}
$$

where the infimum is taken over all paths $\gamma$ joining $x$ and $y$ in $G$. The results of this section are summarized in the following theorem.

Theorem 1.6. For $G \varsubsetneqq \mathbb{R}^{n}$ the following conditions are equivalent:

1. There exists $C>0$ such that $\bar{\alpha}_{G}(x ; r) d(x, \partial G) \geq C$ for all $x \in G$ and $r \in S^{n-1}$;

2. There exists $C>0$ such that $\frac{1}{C} k_{G} \leq \tilde{\alpha}_{G} \leq C k_{G}$;

3. There exists $C>0$ such that $j_{G} \leq C \tilde{\alpha}_{G}$.

The structure of the rest of this paper is as follows. In the next section we review the notation and terminology used. In Section 3 we prove Theorem 1.4 and in Section 4 we use this result to prove Theorema 1.2 and 1.5. In Section 5 we relate the Apollonian metric to the quasihyperbolic metric by means of the concept of quasi-isotropy and prove Theorem 1.6. 


\section{Notation and terminology.}

The notation used conforms largely to that in [2] and [19], as was mentioned in the introduction, and is reviewed in Section 2.1. Sections 2.2 and 2.3, on the other hand, contain material specific to the Apollonian metric.

\subsection{Common notation.}

We denote by $\left\{e_{1}, e_{2}, \ldots, e_{n}\right\}$ the standard basis of $\mathbb{R}^{n}$ and by $n$ the dimension of the Euclidean space under consideration and assume that $n \geq 2$. For $x \in \mathbb{R}^{n}$ we denote by $x_{i}$ its $i^{\text {th }}$ coordinate. The following notation is used for balls, spheres and the upper half-space $\left(x \in \mathbb{R}^{n}\right.$ and $\left.0<r<\infty\right)$ :

$$
\begin{aligned}
B^{n}(x, r) & :=\left\{y \in \mathbb{R}^{n}:|x-y|<r\right\}, S^{n-1}(x, r):=\left\{y \in \mathbb{R}^{n}:|x-y|=r\right\}, \\
B^{n} & :=B^{n}(0,1), S^{n-1}:=S^{n-1}(0,1), H^{n}:=\left\{y \in \mathbb{R}^{n}: y_{n}>0\right\} .
\end{aligned}
$$

We use the notation $\overline{\mathbb{R}^{n}}:=\mathbb{R}^{n} \cup\{\infty\}$ for the one point compactification of $\mathbb{R}^{n}$. If $G \subset \overline{\mathbb{R}^{n}}$ we denote by $\partial G, G^{c}$ and $\bar{G}$ its boundary, complement and closure, respectively, all with respect to $\overline{\mathbb{R}^{n}}$. In contrast to topological operations, we always consider metric operations with respect to the ordinary Euclidean metric. For $x \in G \varsubsetneqq \mathbb{R}^{n}$ we denote $\delta(x):=d(x, \partial G):=\min \{\mid x-$ $z \mid: z \in \partial G\}$.

\subsection{The Apollonian balls approach.}

In this subsection we present the Apollonian balls approach which has previously been used in [3, Section 2], [4] and [17, Theorem 4.1]. This presentation is from Section 5.1 of [11].

For $x, y \in G \varsubsetneqq \mathbb{R}^{n}$ we define

$$
q_{x}:=\sup _{a \in \partial G} \frac{|a-x|}{|a-y|}, q_{y}:=\sup _{b \in \partial G} \frac{|b-y|}{|b-x|} .
$$

The numbers $q_{x}$ are called the Apollonian parameters of $x$ and $y$ (with respect to $G)$ and by definition $\alpha_{G}(x, y)=\log \left\{q_{x} q_{y}\right\}$. The balls or half-spaces

$$
B_{x}:=\left\{z \in \mathbb{R}^{n}: \frac{|z-x|}{|z-y|}<\frac{1}{q_{x}}\right\} \text { and } B_{y}:=\left\{z \in \mathbb{R}^{n}: \frac{|z-y|}{|z-x|}<\frac{1}{q_{y}}\right\},
$$

are called the Apollonian balls about $x$ and $y$ (in $G$ ). We denote the centers and radii of $B_{x}$ and $B_{y}$ by $x_{0}, y_{0}, r_{x}$ and $r_{y}$. We collect some immediate results regarding these balls, similar results obviously hold with $x$ and $y$ interchanged. 
1. $B_{x} \subset G$ and $\overline{B_{x}} \cap \partial G \neq \emptyset$.

2. If $i_{x}$ and $i_{y}$ denote the inversions in the spheres $\partial B_{x}$ and $\partial B_{y}$, then $y=i_{x}(x)=i_{y}(x)$.

3. Since $\infty \notin G$ we have $q_{x} \geq 1$, if moreover $\infty \notin \bar{G}$, then $q_{x}>1$.

4. If $q_{x}>1, x_{0}$ denotes the center of $B_{x}$ and $r_{x}$ its radius, then

$$
\left|x-x_{0}\right|=\frac{|x-y|}{q_{x}^{2}-1}=\frac{r_{x}}{q_{x}} .
$$

\subsection{The Apollonian spheres and quasi-isotropy.}

Let us start by defining the concept of quasi-isotropy, which was introduced [8] and was studied in depth in [10].

Definition 2.1. We say that a metric space $(G, d)$ with $G \subset \mathbb{R}^{n}$ is $K$-quasiisotropic if

$$
\limsup _{r \rightarrow 0} \frac{\sup \{d(x, z):|x-z|=r\}}{\inf \{d(x, y):|x-y|=r\}} \leq K
$$

for every $x \in G$. A 1-quasi-isotropic metric space is called isotropic.

Zair Ibragimov calls a point $x \in G$ for which

$$
\limsup _{r \rightarrow 0} \frac{\sup \left\{\alpha_{G}(x, z):|x-z|=r\right\}}{\inf \left\{\alpha_{G}(x, y):|x-y|=r\right\}}=1
$$

a point of conformality of the Apollonian metric. In [13, Theorem 2] he showed that for the Apollonian metric either none, one or all points are points of conformality. The middle case provides an interesting link to convex sets of constant width, see [13, Theorem 3].

We say that a domain $G \varsubsetneqq \mathbb{R}^{n}$ is quasi-isotropic if $\left(G, \alpha_{G}\right)$ is, similarly for isotropic. We define the function $q i$ on the set of proper subdomains of $\mathbb{R}^{n}$ so that $q i(G)$ is the infimum over constants for which $G$ is quasi-isotropic or $q i(G)=\infty$ if $G$ is not quasi-isotropic for any $K$.

Recall that the density of the Apollonian metric was defined in the introduction. With this concept we can give the following alternative characterization of quasi-isotropy.

Lemma 2.2 (Lemma 3.5, [10]). For $G \varsubsetneqq \mathbb{R}^{n}$ we have

$$
q i(G)=\sup _{x \in G} \frac{\sup _{r \in S^{n-1}} \bar{\alpha}_{G}(x ; r)}{\inf _{r \in S^{n-1}} \bar{\alpha}_{G}(x ; r)},
$$


with the understanding that if $\bar{\alpha}_{G}(x ; r)=0$ for some $x \in G$ and $r \in S^{n-1}$ then $q i(G)=\infty$.

When we do not need the exact value of the quasi-isotropy constant the following lemma is often more convenient to use.

Lemma 2.3 (Lemma 4.2, [8]). Let $G \varsubsetneqq \mathbb{R}^{n}$ be $L$-quasi-isotropic. Then $\bar{\alpha}_{G}(x ; r) \delta(x) \geq 1 / L$ for every $x \in G$ and $r \in S^{n-1}$.

If conversely $\bar{\alpha}_{G}(x ; r) \delta(x) \geq 1 / L$ for every $x \in G$ and $r \in S^{n-1}$, then $G$ is $2 L$-quasi-isotropic.

\section{An integral formula for the Apollonian inner metric.}

In this section we prove that we can evaluate the Apollonian inner metric by taking the integral over the density on piecewise continuously differentiable paths.

The idea with the integral formulae for inner metrics is that the density is the derivative of the metric considered as a function of one variable and the inner metric is essentially the Riemann integral sum over this derivative. In fact for continuously differentiable paths this is strictly speaking true both for the $j_{G}$ and the Apollonian metric (the latter claim is proved in Lemma 3.2). Since the density of the $j_{G}$ metric, $1 / \delta(x)$, does not depend on the direction we can then use a density argument of continuously differentiable paths in the set of all paths to conclude that $\tilde{j}_{G}=k_{G}$.

For the Apollonian metric, whose density depends on the direction, we would have to approximate not only a path but also its derivative, however, the latter need not even be defined for an arbitrary path. In view of this difficulty, we will show explicitly that Apollonian length of a path is about the same as the Apollonian length of a piecewise linear approximation of the path. This will be done in two steps, first for quasi-isotropic domains in Lemma 3.5 and then for general domains in Corollary 3.11. With this sketch of the proof in mind we proceed with the details.

Unless specified to the contrary we assume throughout this paper that every path $\gamma$ is defined on the set $[0,1]$ and we call a sequence $\left\{t_{i}\right\}_{i=0}^{k}$ with $0=t_{0}<t_{1}<\ldots<t_{k}=1$ a length sequence. Let us denote, for the time being,

$$
\tilde{\alpha}_{G}^{\prime}(\gamma)=\int_{0}^{1} \bar{\alpha}_{G}\left(\gamma(t) ; \gamma^{\prime}(t)\right)\left|\gamma^{\prime}(t)\right| d t
$$

where $\gamma \subset G$ is a piecewise differentiable (as before, we define $\bar{\alpha}_{G}(x ; 0) 0=0$ ). We start by showing that the integral formula is valid for segments. 
Many of the arguments that follow are based on the fact that " $\alpha_{G}$ is continuous" which is an immediate consequence of the definition of the metric.

Lemma 3.2. For every segment $[x, y] \subset G$ we have $\alpha_{G}([x, y])=\tilde{\alpha}_{G}^{\prime}([x, y])$.

Proof. We may obviously assume that $x \neq y$. Denote $r:=y-x$ and choose $\gamma(t):=x+r t$, from which it follows that the kernel of the integral in (3.1) equals $\bar{\alpha}_{G}(x+r t ; r)$. Since $\alpha_{G}(x, x+r t) / t$ is continuous and tends to $\bar{\alpha}_{G}(x ; r)$ as $t \rightarrow 0$ (shown in Lemma 5.8, [8]) and since $[x, y]$ is compact we may, for $\epsilon>0$, choose $\delta>0$ such that

$$
\alpha_{G}(z, w) /(1+\epsilon) \leq \bar{\alpha}_{G}(z ; r)|z-w| \leq(1+\epsilon) \alpha_{G}(z, w)
$$

for $z, w \in[x, y]$ with $|z-w|<\delta$. Moreover, there exists a $\delta^{\prime}>0$ such that

$$
\left|\int_{0}^{1} \bar{\alpha}_{G}(x+r t ; r)\right| r\left|d t-\sum_{i=0}^{k-1} \bar{\alpha}_{G}\left(x+r t_{i} ; r\right)\right| r|| t_{i+1}-t_{i}|| \leq \epsilon,
$$

for every length sequence $\left\{t_{i}\right\}$ satisfying $t_{i+1}-t_{i}<\delta^{\prime}$ for all $0 \leq i<k$, since $z \mapsto \bar{\alpha}_{G}(z ; r)$ is continuous and hence integrable. Taking the supremum over all length sequences with

$$
\max \left\{t_{i+1}-t_{i}\right\} \leq \min \left\{\delta^{\prime}, \delta /|r|\right\}
$$

and using the first inequality of (3.3) we find that

$$
\begin{aligned}
\alpha_{G}([x, y]) /(1+\epsilon) & \leq \sup \sum_{i=0}^{k-1} \bar{\alpha}_{G}\left(x+r t_{i} ; r\right)|r|\left|t_{i+1}-t_{i}\right| \\
& \leq \int_{0}^{1} \bar{\alpha}_{G}(x+r t ; r)|r| d t+\epsilon
\end{aligned}
$$

Since $\epsilon$ was arbitrary, the inequality $\alpha_{G}([x, y]) \leq \tilde{\alpha}_{G}^{\prime}([x, y])$ is thus proved. The converse inequality follows similarly.

Remark 3.4. The previous proof also shows that the integral formula holds for paths that are continuously differentiable almost everywhere, in particular for piecewise linear paths.

We now proceed to the harder part of the proof - showing that we do not gain anything by allowing (say) widely oscillating nondifferentiable paths in the infimum. We first consider domains in which the Apollonian metric is quasi-isotropic. The intuitive reason for this restriction is that we can then estimate the Apollonian metric in a uniform manner. 
Lemma 3.5. Let $G$ be $L$-quasi-isotropic and $x, y \in G$. Then

$$
\inf _{\gamma \in A} \alpha_{G}(\gamma)=\inf _{\gamma \in B} \alpha_{G}(\gamma)
$$

where $A$ is the set of all paths joining $x$ and $y$ in $G$ and $B$ is the set of all piecewise linear paths joining $x$ and $y$ in $G$.

Proof. Since $B \subset A$, it is clear that

$$
\inf _{\gamma \in A} \alpha_{G}(\gamma) \leq \inf _{\gamma \in B} \alpha_{G}(\gamma)
$$

so it remains only to prove the opposite inequality. Fix $\gamma \in A$. We will show that for every $\epsilon>0$ there exists a length sequence such that we have

$$
(1+\epsilon) \alpha_{G}\left(\gamma\left(t_{i}\right), \gamma\left(t_{i+1}\right)\right) \geq \alpha_{G}\left(\left[\gamma\left(t_{i}\right), \gamma\left(t_{i+1}\right)\right]\right)
$$

for every $0 \leq i<k$. Adding these inequalities we see that

$$
\begin{aligned}
(1+\epsilon) \alpha_{G}(\gamma) & \geq(1+\epsilon) \sum_{i=0}^{k-1} \alpha_{G}\left(\gamma\left(t_{i}\right), \gamma\left(t_{i+1}\right)\right) \\
& \geq \sum_{i=0}^{k-1} \alpha_{G}\left(\left[\gamma\left(t_{i}\right), \gamma\left(t_{i+1}\right)\right]\right)=\alpha_{G}\left(\gamma^{\prime}\right),
\end{aligned}
$$

where $\gamma^{\prime}$ is the path formed by concatenating the segments $\left[\gamma\left(t_{i}\right), \gamma\left(t_{i+1}\right)\right]$. Since $\epsilon$ was arbitrary, this shows that the opposite inequality holds too.

It remains to construct a length sequence such that (3.6) holds. Let us denote $d(\gamma, \partial G)=: c>0$. We will show that there exists a constant $u \in(0,0.15)$ depending on $L$ and $\epsilon$ such that

$$
(1+\epsilon) \alpha_{G}(x, y) \geq \alpha_{G}([x, y])
$$

for every $x, y \in \gamma$ with $|x-y| \leq u c$. It follows from this that it suffices to choose $\left\{t_{i}\right\}_{i=0}^{k}$ to be some sequence with $\left|\gamma\left(t_{i}\right)-\gamma\left(t_{i+1}\right)\right| \leq u c$ for all $0 \leq i<k$, which is possible (for some $k$ ), since $\gamma$ is rectifiable.

Let us assume then that $x, y \in \gamma$ with $|x-y| \leq u c$. Let $B_{x}$ and $B_{y}$ be the Apollonian balls about $x$ and $y$ and let $q_{x}$ and $q_{y}$ be the Apollonian parameters. We note that

$$
\max \left\{q_{x}, q_{y}\right\} \leq q_{x} q_{y}=\exp \alpha_{G}(x, y) \leq \exp \left\{2 j_{G}(x, y)\right\} \leq(1+u)^{2} \leq 1.4,
$$

where the second inequality is from [3, Theorem 3.2]. 


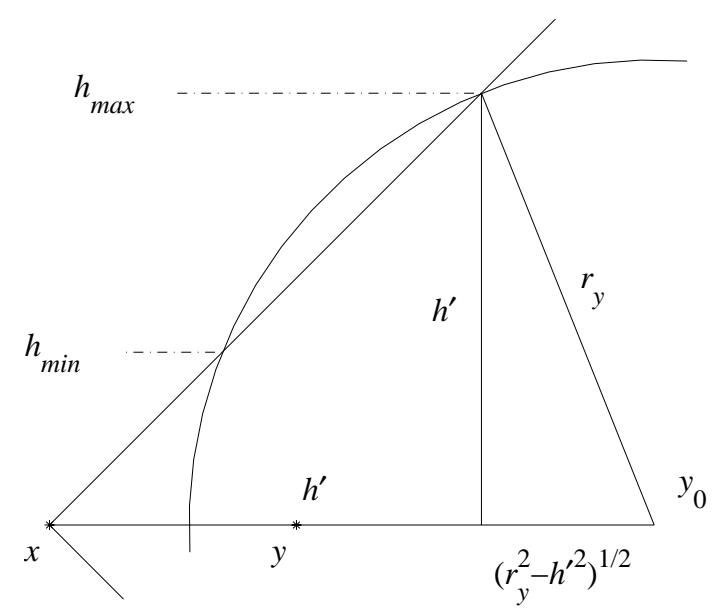

Figure 1: Calculating $h$ when the mantle of the cylinder is the limiting factor.

It is clear that the balls $B_{x}, B_{y}, B^{n}(x, c)$ and $B^{n}(y, c)$ all lie in $G$. Define $h:=\min \left\{r_{y}, \sqrt{c^{2}-|x-y|^{2} / 4}\right\}$ and let $C$ be the cylinder around the segment $\left[x_{0}, y_{0}\right]$ (recall that $x_{0}$ is the center of $B_{x}$ and $y_{0}$ of $B_{y}$ ) with radius $h$. We easily see that

$$
G^{\prime}:=\left(B_{x} \cup B_{y} \cup C\right) \subset\left(B_{x} \cup B_{y} \cup B^{n}(x, c) \cup B^{n}(y, c)\right) \subset G
$$

(the domain $G^{\prime}$ is indicated in Figure 2 by the heavy line). Since the Apollonian spheres about $x$ and $y$ are the same in $G$ and $G^{\prime}$ we find that $\alpha_{G}(x, y)=$ $\alpha_{G^{\prime}}(x, y)=\log \left\{q_{x} q_{y}\right\}$. Since the Apollonian metric is monotone in the domain of definition (see $\left[3\right.$, Introduction (3)]) we have $\alpha_{G}([x, y]) \leq \alpha_{G^{\prime}}([x, y])$ and thus it suffices to show that $(1+\epsilon) \alpha_{G^{\prime}}(x, y) \geq \alpha_{G^{\prime}}([x, y])$ in order to prove (3.7).

Let $z \in[x, y]$ and define $t:=|y-z|$. The Apollonian sphere in $G^{\prime}$ through $z$ in direction $y-x$ is limited by either the mantel of $C \backslash B_{y}$ or by $(\partial C) \cap\left(\partial B_{y}\right)$ when $z \notin B_{y}$ and by the sphere $\partial B_{y}$ when $z \in B_{y}$. We will derive an estimate for the radius of the Apollonian sphere in each case.

Suppose first that the Apollonian sphere through $z$ in direction $y-x$ is limited by the mantel of $C \backslash B_{y}$ for some $z$. It is clear that so is the Apollonian sphere through $x$ in the same direction. This means that the cone with vertex $x$, direction $y-x$ and angle $\pi / 4$ intersects the mantel of $C$. We calculate where this cone intersects $\partial B_{y}$. Let $h^{\prime}$ denote the distance of the points of intersection from the line $x y$. We have $h^{\prime}+\sqrt{r_{y}^{2}-h^{\prime 2}}=\left|x-y_{0}\right|=q_{y} r_{y}$, see 
Figure 1. This second degree equation in $h^{\prime}$ has roots (we used the formulae from observation 4 in Section 2.2)

$$
h^{\prime}=\frac{r_{y}}{2}\left(q_{y} \pm \sqrt{2-q_{y}^{2}}\right) .
$$

Since this cone is supposed to intersect the mantel of $C$ in $B_{y}^{c}$ it is clear that either $h$ (the radius of the cone) is smaller than the smaller root or larger than the larger one. Consider first the smaller of root. We find

$$
h_{\text {min }}=\frac{|x-y|}{2} \frac{q_{y}}{q_{y}^{2}-1} \frac{q_{y}^{2}-\left(2-q_{y}^{2}\right)}{q_{y}+\sqrt{2-q_{y}^{2}}}=\frac{q_{y}}{q_{y}+\sqrt{2-q_{y}^{2}}}|x-y|
$$

and we see that this root is smaller than $|x-y|$. On the other hand we have

$$
\begin{aligned}
h=\min \left\{r_{y}, \sqrt{c^{2}-|x-y|^{2} / 4}\right\} & \geq \min \left\{\frac{|x-y|}{q_{y}-1 / q_{y}},|x-y| \sqrt{u^{-2}-1}\right\} \\
& \geq \min \{1.45,6.5\}|x-y|,
\end{aligned}
$$

where we used $q_{y} \leq 1.4$ and $u \leq 0.15$. Hence we see that $h$ is not smaller than the smaller root, and so we conclude that it is greater than the larger root, which we denote by $h_{\max }$.

Suppose next that $z$ is such that the Apollonian sphere through $z$ in direction $y-x$ is limited by $(\partial C) \cap\left(\partial B_{y}\right)$. With the variables indicated in Figure 2 we find the radius of this sphere equals $w /(2 \cos \theta)=w^{2} /(2 s)$, where $s:=t+r_{y} / q_{y}-\sqrt{r_{y}^{2}-h^{2}}, w:=\sqrt{s^{2}+h^{2}}$ and $\cos \theta:=s / w$. Using the expressions for $s$ and $w$ we find that

$$
\begin{aligned}
\frac{s}{w^{2}} & =\frac{t+r_{y} / q_{y}-\sqrt{r_{y}^{2}-h^{2}}}{\left(t+r_{y} / q_{y}-\sqrt{r_{y}^{2}-h^{2}}\right)^{2}+h^{2}} \\
& \leq \frac{|x-y|}{\left(\frac{r_{y}}{q_{y}}-\sqrt{r_{y}^{2}-h^{2}}\right)^{2}+h^{2}}+\frac{r_{y}-\sqrt{r_{y}^{2}-h^{2}}}{\left(r_{y}-\frac{r_{y}}{q_{y}}\right)^{2}+\frac{2 r_{y}}{q_{y}}\left(r_{y}-\sqrt{r_{y}^{2}-h^{2}}\right)} \\
& \leq \frac{|x-y|}{h^{2}}+\frac{q_{y}}{2 r_{y}} .
\end{aligned}
$$

Here we used $t \leq|x-y|$ and $r_{y} / q_{y} \leq r_{y}$ in the first inequality and threw away some positive terms from the denominators for the second inequality.

Finally, in the case when the Apollonian sphere through $z$ in direction $y-x$ is limited by $\partial B_{y}$ we find that its radius equals $\left(r_{y}+\left|y_{0}-z\right|\right) / 2 \geq$ $\left(r_{y}+\left|y_{0}-y\right|\right) / 2=r_{y}\left(1+1 / q_{y}\right) / 2$. 


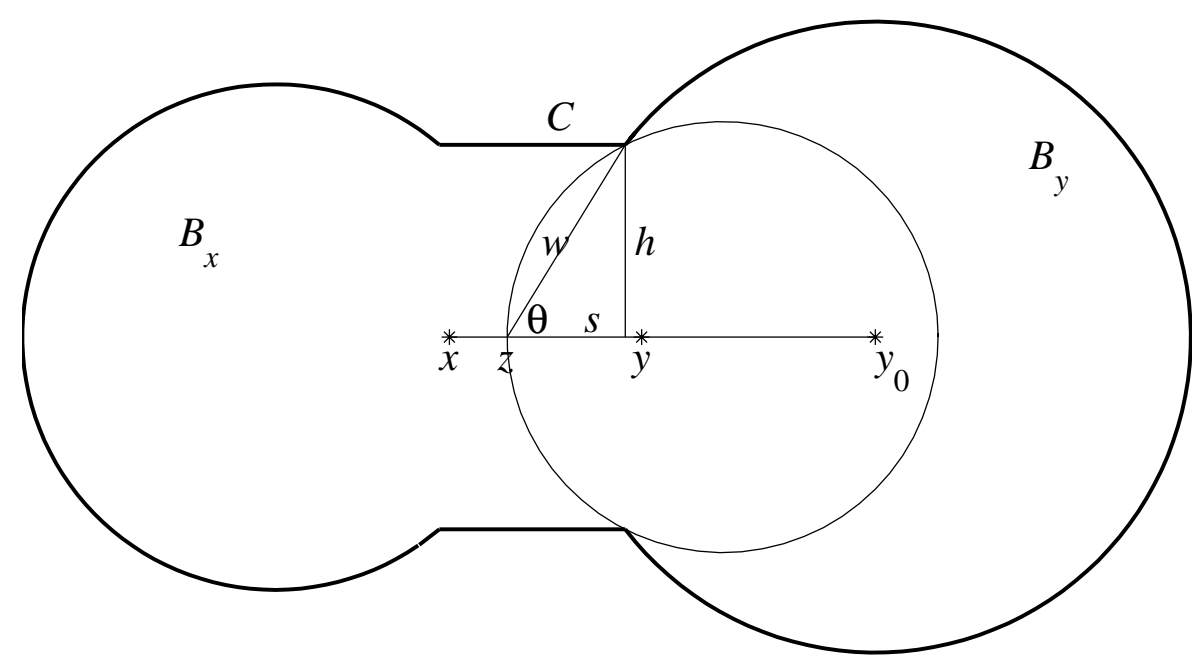

Figure 2: Calculating the radius of the Apollonian sphere when $(\partial C) \cap\left(\partial B_{y}\right)$ is the limiting factor.

Let $r_{+}$denote the radius of the Apollonian sphere thorough $z$ in direction $y-x$. Combining the estimates from the previous three paragraphs we see that

$$
\begin{aligned}
\frac{1}{2 r_{+}} & \leq \max \left\{\frac{1}{2 h_{\max }}, \frac{s}{w^{2}}, \frac{1}{r_{y}+\left|y_{0}-z\right|}\right\} \\
& \leq \max \left\{\frac{1}{\left(q_{y}+\sqrt{2-q_{y}^{2}}\right) r_{y}}, \frac{q_{y}}{2 r_{y}}+\frac{|x-y|}{h^{2}}, \frac{1}{\left(1+1 / q_{y}\right) r_{y}}\right\} \\
& =\frac{q_{y}}{2 r_{y}}+\frac{|x-y|}{h^{2}} .
\end{aligned}
$$

Similarly we derive an estimate for the radius $r_{-}$of the Apollonian sphere through $z$ in direction $x-y$ and find that

$$
\frac{1}{2 r_{-}} \leq \frac{q_{x}}{2 r_{x}}+\frac{|x-y|}{h_{x}^{2}}
$$

where $h_{x}:=\min \left\{r_{x}, \sqrt{c^{2}-|x-y|^{2} / 4}\right\}$. 
It follows from these estimates and Lemma 3.2 that

$$
\begin{aligned}
\alpha_{G}([x, y]) & =\int_{[x, y]} \frac{1}{2 r_{+}}+\frac{1}{2 r_{-}} d s \\
& \leq \frac{q_{x}|x-y|}{2 r_{x}}+\frac{q_{y}|x-y|}{2 r_{y}}+2 \rho^{2} \\
& =q_{x}^{2} / 2+q_{y}^{2} / 2-1+2 \rho^{2},
\end{aligned}
$$

where we have denoted $\rho:=|x-y| / m$ for $m:=\min \left\{r_{x}, r_{y}, \sqrt{c^{2}-|x-y|^{2} / 4}\right\}$. We have now shown that

$$
\frac{\alpha_{G}([x, y])}{\alpha_{G}(x, y)} \leq \frac{q_{x}^{2} / 2+q_{y}^{2} / 2-1+2 \rho^{2}}{\log \left\{q_{x} q_{y}\right\}}
$$

We next show that we can choose $u$ so that the left hand side of the previous inequality is bounded from above by $1+\epsilon$.

We start by deriving a bound for $\rho$. Since $G$ is $L$-quasi-isotropic we have, by [8, Lemma 4.5],

$$
\log \left\{1+|x-y| /\left(c L^{\prime}\right)\right\} \leq j_{G}(x, y) / L^{\prime} \leq \alpha_{G}(x, y)=\log \left\{q_{x} q_{y}\right\},
$$

where $L^{\prime}$ depends on $L$ and $c$. If $m=\sqrt{c^{2}-|x-y|^{2} / 4}$ this implies that

$$
\rho=\frac{|x-y|}{\sqrt{c^{2}-|x-y|^{2} / 4}} \leq \frac{|x-y| / c}{\sqrt{1-u^{2} / 4}} \leq 1.1 \frac{|x-y|}{c} \leq 1.1 L^{\prime}\left(q_{x} q_{y}-1\right) .
$$

In case $m=r_{x}$, we have $\rho=q_{x}-1 / q_{x} \leq 2\left(q_{x}-1\right)$ and for $m=r_{y}$ we have $\rho \leq 2\left(q_{y}-1\right)$. Hence in every case $\rho \leq 2 L^{\prime}\left(q_{x} q_{y}-1\right)$. We then find that

$$
\frac{2 \rho^{2}}{\log \left\{q_{x} q_{y}\right\}} \leq \frac{8 L^{\prime 2}\left(q_{x} q_{y}-1\right)^{2}}{\log \left\{q_{x} q_{y}\right\}} \leq \frac{72 L^{\prime 2} u^{2}}{\log \{1+3 u\}},
$$

where we used $q_{x} q_{y} \leq(1+u)^{2} \leq 1+3 u$ and the fact that $z \mapsto z^{2} / \log \{1+z\}$ is increasing in $z$ for the second inequality. Since $72 L^{\prime 2} u^{2} / \log \{1+3 u\} \rightarrow 0$ as $u \rightarrow 0$, we can choose $u_{0}>1$ so that $72 L^{\prime 2} u^{2} / \log \{1+3 u\} \leq \epsilon / 2$ for $u<u_{0}$.

We move on to the other terms. Since

$$
\lim _{q_{x} \rightarrow 1+} \frac{q_{x}^{2}-1}{2 \log q_{x}}=1
$$

there exists a $q>1$ such that $q_{x}^{2}-1 \leq 2(1+\epsilon / 2) \log q_{x}$ holds for $q_{x}<q$. Then for $u<\min \left\{\sqrt{q}-1, u_{0}\right\}$ it follows that $\max \left\{q_{x}, q_{y}\right\} \leq(1+u)^{2}<q$, 
and so we have

$$
\begin{aligned}
\frac{\left(q_{x}^{2}-1\right) / 2+\left(q_{y}^{2}-1\right) / 2+2 \rho^{2}}{\log \left\{q_{x} q_{y}\right\}} & \leq \frac{(1+\epsilon / 2)\left(\log q_{x}+\log q_{y}\right)}{\log \left\{q_{x} q_{y}\right\}}+\frac{72 L^{\prime 2} u^{2}}{\log \{1+3 u\}} \\
& \leq 1+\epsilon,
\end{aligned}
$$

which completes the proof.

In the previous proof the claim that every length sequence with $\mid \gamma\left(t_{i}\right)-$ $\gamma\left(t_{i+1}\right) \mid<u c$ satisfies (3.7) is false if we drop the assumption that the domain is quasi-isotropic. On the other hand, (3.7) holds for "most" points even in this case, a fact that we will exploit. We develop a method for removing the assumption of quasi-isotropy, which is based on constructing a sequence of domains approaching a given domain with larger and larger quasi-isotropy constants.

Definition 3.8. For $G \varsubsetneqq \mathbb{R}^{n}$ we define $G_{r}:=\{x \in G: d(x, \partial G)>r\}$.

Lemma 3.9. Let $G \varsubsetneqq \mathbb{R}^{n}$ be a domain and $x \in G$. For every $r>0$ and $R>0$ the set $G_{r} \cap B^{n}(x, R)$ is quasi-isotropic.

Proof. We have

$$
G_{r}=G \backslash \bigcup_{z \in \partial G} \overline{B^{n}}(z, r),
$$

hence for every boundary point $w$ of $G_{r}$ there exists a ball $B^{n}(z, r)$ which is not in $G_{r}$ for which $w \in S^{n-1}(z, r)$. This is clearly also true for all boundary points of $G_{r} \cap B^{n}(x, R)$. Hence it follows from [11, Theorem 4.3] that this domain has the approximation property (the ball condition derived above is called the $(1, r)$-EB condition in that paper) and is therefore quasi-isotropic.

Lemma 3.10. Let $x, y \in G \varsubsetneqq \mathbb{R}^{n}$ and $\gamma_{0}$ be an $\alpha_{G}$-rectifiable path connecting $x$ and $y$. Define $G^{\prime}:=G_{r} \cap B^{n}(x, R)$ where $c:=\alpha_{G}\left(\gamma_{0}\right), r:=\delta(x) /\left(e^{c}+2\right)$ and $R:=\left(e^{c}+1\right) \delta(x)$. Then

$$
\inf _{\gamma \in A} \alpha_{G}(\gamma)=\inf _{\gamma \in B} \alpha_{G}(\gamma),
$$

where $A$ is the set of all paths joining $x$ and $y$ in $G$ and $B$ is the set of all paths joining $x$ and $y$ in $G^{\prime}$. 
Proof. If $\gamma$ is a path connecting $x$ to a point $z \in G$ with $\delta(z)<\delta(x) / 3$, then

$$
\alpha_{G}(\gamma) \geq \alpha_{G}(x, z) \geq \log \{|x-z| / \delta(z)-1\} \geq \log \{\delta(x) / \delta(z)-2\},
$$

as we see by choosing $a=\infty$ and $b$ such that $|z-b|=\delta(z)$ in the supremum in the definition of the Apollonian metric for a lower bound. If $\delta(z)<$ $\delta(x) /\left(e^{c}+2\right)$ then this means that $\alpha_{G}(\gamma)>c$. Let next $\gamma$ be a path such that $\gamma \cap S^{n-1}(x, R) \neq \emptyset$ for some $R>3 \delta(x)$ and let $z \in \gamma \cap S^{n-1}(x, R)$. Then we have

$$
\alpha_{G}(\gamma) \geq \alpha_{G}(x, z) \geq \log \{|x-z| / \delta(x)-1\}
$$

Now if $|x-z|=R>\left(e^{c}+1\right) \delta(x)$, then again $\alpha_{G}(\gamma)>c$. We have thus seen that if $\gamma \in A \backslash B$, then $\alpha_{G}(\gamma)>c$ and so we see that such paths have no bearing on the infimum which is less than or equal to $c$, anyway.

Using these auxiliary lemmata we show that the assumption of quasiisotropy was not essential in Lemma 3.5.

Corollary 3.11. For every domain $G \varsubsetneqq \mathbb{R}^{n}$ we have

$$
\inf _{\gamma \in A} \alpha_{G}(\gamma)=\inf _{\gamma \in C} \alpha_{G}(\gamma)
$$

where $A$ is the set of all paths joining $x$ and $y$ in $G$ and $C$ is the set of all piecewise linear paths joining $x$ and $y$ in $G$.

Proof. Since $C \subset A$ we have

$$
\inf _{\gamma \in A} \alpha_{G}(\gamma) \leq \inf _{\gamma \in C} \alpha_{G}(\gamma)
$$

and so it remains only to consider the opposite inequality.

Let $x \in G$ and define $G(c):=G_{c} \cap B^{n}(x, 1 / c)$. Using Lemma 3.9, we see that $G(c)$ is quasi-isotropic for $c>0$. Since $\tilde{\alpha}_{G} \leq 2 k_{G}$ by [3, Theorem 3.2], we see that there exists a path connecting $x$ and $y$ in $G$ which is $\tilde{\alpha}_{G}$-rectifiable. Let $\gamma_{0}$ be the one such path and let $G^{\prime}$ be as in Lemma 3.10 and define $K:=\overline{G^{\prime}}$. Since $\alpha_{G}(x, y)$ is continuous in the domain of definition $G$, it follows that for every $\epsilon>0$ there exists a $c_{0}>0$ such that

$$
\alpha_{G(c)}(z, w) /(1+\epsilon) \leq \alpha_{G}(z, w)
$$


holds for all $z, w \in K$ and every $c<c_{0}$. Let $B$ be as in Lemma 3.10. We have

$$
\begin{aligned}
\inf _{\gamma \in A} \alpha_{G}(\gamma)=\inf _{\gamma \in B} \alpha_{G}(\gamma) & \geq \frac{1}{1+\epsilon} \inf _{\gamma \in B} \alpha_{G(c)}(\gamma)=\frac{1}{1+\epsilon} \inf _{\gamma \in C} \alpha_{G(c)}(\gamma) \\
& \geq \frac{1}{1+\epsilon} \inf _{\gamma \in C} \alpha_{G}(\gamma) .
\end{aligned}
$$

Here the first equality follows from Lemma 3.10, the first inequality from (3.12), the second equality from Lemma 3.5 and the last inequality since $\alpha_{G}$ is monotone in the domain of definition by [3, Introduction (3)]. Since $\epsilon$ can be arbitrarily small this implies that $\inf _{\gamma \in A} \alpha_{G}(\gamma) \geq \inf _{\gamma \in C} \alpha_{G}(\gamma)$, which was to be shown.

Proof of Theorem 1.4. Fix $x, y \in G$ and let $A$ be the set of all paths joining $x$ and $y$ in $G, B$ be the set of piecewise linear paths in $A$ and $C$ be the set of paths in $A$ which are piecewise continuously differentiable. It is clear that $B \subset C \subset A$ and so

$$
\inf _{\gamma \in A} \alpha_{G}(\gamma) \leq \inf _{\gamma \in C} \alpha_{G}(\gamma) \leq \inf _{\gamma \in B} \alpha_{G}(\gamma)
$$

By Corollary 3.11 we have $\inf _{\gamma \in A} \alpha_{G}(\gamma)=\inf _{\gamma \in B} \alpha_{G}(\gamma)$, which combined with the previous inequality gives

$$
\inf _{\gamma \in A} \alpha_{G}(\gamma)=\inf _{\gamma \in C} \alpha_{G}(\gamma)=\inf _{\gamma \in B} \alpha_{G}(\gamma)
$$

Finally, it follows from Remark 3.4 that

$$
\inf _{\gamma \in C} \tilde{\alpha}_{G}(\gamma)=\inf _{\gamma \in C} \tilde{\alpha}_{G}^{\prime}(\gamma)
$$

which concludes the proof.

\section{Pseudometrics and geodesics.}

In this section we consider two basic aspects of the Apollonian inner metric, namely, in which domains it is a metric and in which domains there exist geodesics connecting arbitrary points.

Proof of Theorem 1.2. Since $\alpha_{G}$ satisfies the triangle inequality, is nonnegative and symmetric it is clear that so does and is $\tilde{\alpha}_{G}$. It thus remains to 
consider whether or not the Apollonian inner metric is positive definite for a given domain, i.e whether it is a pseudometric or a metric.

Suppose first that $G^{c}$ is contained in an $(n-2)$-dimensional plane, and assume without loss of generality that this plane is $\left\{x \in \mathbb{R}^{n}: x_{n-1}=x_{n}=0\right\}$. Define $\gamma(t)=\sin (\pi t) e_{n-1}+\cos (\pi t) e_{n}$ for $t \in[0,1]$. For every $x, y \in \gamma([0,1])$ we have $\alpha_{G}(x, y)=0$ since every boundary point of $G$ is at an equal distance from $x$ and $y$. This means that $\alpha_{G}(\gamma)=0$ and hence $\tilde{\alpha}_{G}\left(e_{n},-e_{n}\right)=0$ which means that $\tilde{\alpha}_{G}$ is a pseudometric.

It remains to check that for distinct $x, y \in G$ we have $\tilde{\alpha}_{G}(x, y)>0$ if $G^{c}$ is not contained in an $(n-2)$-dimensional plane. If $G^{c}$ is not contained in a hyperplane, then $\alpha_{G}$ is a metric and it is clear that so is $\tilde{\alpha}_{G}$, since $\tilde{\alpha}_{G}(x, y) \geq \alpha_{G}(x, y)$ always holds. It remains to consider the case when $G^{c}$ is contained in a hyperplane but not in an $(n-2)$-dimensional plane. For definiteness say $\partial G \subset \partial H^{n}$. It is clear that we need only consider the Apollonian inner metric for distinct $x, y \in G$ such that $\alpha_{G}(x, y)=0$, which means that $x$ and $y$ are each others mirror images in the plane $\partial H^{n}$. Assume without loss of generality that $x_{n}>0$. This means that $\bar{\alpha}_{G}(z ; \theta)>0$ for every $z \in \overline{B^{n}}\left(x, x_{n} / 2\right)=: B$ and $\theta \in S^{n-1}$, since $\bar{\alpha}_{G}(z ; \theta)=0$ implies that $\partial G$ is contained in the hyperplane $P$ through $z$ with $\theta$ as normal, which would imply that $\partial G$ would be contained in the $(n-2)$-dimensional plane $P \cap \partial H^{n}$, contrary to assumption. Thus

$$
\bar{\alpha}_{G}(z ; \theta) \geq \min _{z \in B} \min _{\theta \in S^{n-1}} \bar{\alpha}_{G}(z ; \theta)=: a>0
$$

and so $\tilde{\alpha}_{G}(x, y) \geq a x_{n}>0$ since every path connecting $x$ and $y$ has Euclidean length at least $x_{n} / 2$ in $B$ and another $x_{n} / 2$ in $\overline{B^{n}}\left(y, x_{n} / 2\right)$.

We then move on to consider the geodesics.

Proof of Theorem 1.5. Fix $x, y \in G$ and consider the set $A$ of piecewise linear paths connecting $x$ and $y$ in $G$. Since $\tilde{\alpha}_{G} \leq 2 k_{G}$ by [3, Theorem 3.2], we see that there exists a path in $A$ which is $\tilde{\alpha}_{G}$-rectifiable. Let $c_{0}$ be the length of one such path.

Let us define $r:=\delta(x) /\left(e^{c_{0}}+2\right), R:=\left(e^{c_{0}}+1\right) \delta(x), G^{\prime}:=G_{r} \cap B^{n}(x, R)$ and $K:=\overline{G^{\prime}}$. It follows from Lemma 3.10 that we need only consider paths connecting $x$ and $y$ in $K$. Since $\bar{\alpha}_{G}(x ; \theta)>0$ (here we use that $\alpha_{G}$ is a metric) and continuous in $x \in G$ and $\theta \in S^{n-1}$, it follows that it has a minimum in the compact set $K \times S^{n-1}$, say $c_{1}$, and so we see that we need only consider paths of Euclidean length $\ell(\gamma) \leq c_{0} / c_{1}$, since for longer paths we have $\alpha_{G}(\gamma)>\ell(\gamma) c_{1}>c_{0}$. Let $B$ be the set of piecewise linear paths 
connecting $x$ and $y$ in $K$ of Euclidean length at most $c_{0} / c_{1}$.

We have shown that

$$
\inf _{\gamma \in A} \alpha_{G}(\gamma)=\inf _{\gamma \in B} \alpha_{G}(\gamma)
$$

and so we can choose a sequence $\left(\gamma_{i}^{\prime}\right)$ of paths in $B$ parameterized by arclength such that $\lim _{i \rightarrow \infty} \alpha_{G}\left(\gamma_{i}^{\prime}\right)=\tilde{\alpha}_{G}(x, y)$. Define the path $\gamma_{i}$ by $\gamma_{i}(t):=$ $\gamma_{i}^{\prime}\left(t \ell\left(\gamma_{i}^{\prime}\right)\right)$ for $t \in[0,1]$ and every $i$. It is clear that $\lim _{i \rightarrow \infty} \alpha_{G}\left(\gamma_{i}\right)=\tilde{\alpha}_{G}(x, y)$, since the parameterization of the path does not affect its length.

Let next $f: \mathbb{N} \rightarrow[0,1] \cap \mathbb{Q}$ be a surjection. Since $\gamma_{i}(f(0))$ is a sequence of points in the compact set $K$ it follows that there exists a subsequence $\left(\gamma_{i}^{0}\right)$ of $\left(\gamma_{i}\right)$ such that $\gamma_{i}^{0}(f(0))$ converges to a point in $K$. Continuing like this we construct a subsequence $\left(\gamma_{i}^{k}\right)$ of $\left(\gamma_{i}^{k-1}\right)$ such that $\gamma_{i}^{k}(f(j))$ converges for $0 \leq j \leq k$. Let then $\gamma_{\infty}=\lim _{k \rightarrow \infty} \gamma_{k}^{k}$. For every $i$ we have

$$
\left|\gamma_{i}(t)-\gamma_{i}(s)\right| \leq \ell\left(\gamma_{i}\right)|s-t| \leq c_{0}|s-t| / c_{1},
$$

for $s, t \in[0,1]$, where the first inequality follows since the mapping $\gamma_{i}^{\prime}$ is a contraction and the second one since we are considering paths of length less than or equal to $c_{0} / c_{1}$. Hence we see that the family $\left\{\gamma_{i}\right\}$ is equicontinuous which implies that $\gamma_{\infty}$ is continuous and hence a path connecting $x$ and $y$ in $K$.

Suppose then that $\alpha_{G}\left(\gamma_{\infty}\right)-\inf _{\gamma \in A} \alpha_{G}(\gamma)=: \delta>0$. Let $\left\{t_{i}\right\}$ be a sequence with $0=t_{0}<\ldots<t_{l}=1$ and $t_{i} \in \mathbb{Q}$ such that

$$
\sum_{i=0}^{l-1} \alpha_{G}\left(\gamma_{\infty}\left(t_{i+1}\right), \gamma_{\infty}\left(t_{i}\right)\right)>\alpha_{G}\left(\gamma_{\infty}\right)-\delta / 2 .
$$

Let us denote $m:=\inf _{\gamma \in A} \alpha_{G}(\gamma)$ and set $\epsilon:=\delta /(4 m+\delta)$. Let $r_{i}>0$ be such that

$$
\alpha_{G}\left(\gamma_{\infty}\left(t_{i+1}\right), \gamma_{\infty}\left(t_{i}\right)\right)<(1+\epsilon) \alpha_{G}(z, w)
$$

for $z \in B^{n}\left(\gamma_{\infty}\left(t_{i+1}\right), r_{i}\right)$ and $w \in B^{n}\left(\gamma_{\infty}\left(t_{i}\right), r_{i}\right)$ and define $r:=\min r_{i}$. Since $\gamma_{k}^{k}\left(t_{i}\right) \rightarrow \gamma_{\infty}\left(t_{i}\right)$ we can find a $k_{0}$ such that $\left|\gamma_{k}^{k}\left(t_{i}\right)-\gamma_{\infty}\left(t_{i}\right)\right|<r$ for all $k>k_{0}$ and all $i$. It then follows that for $k>k_{0}$ we have

$$
\begin{aligned}
\alpha_{G}\left(\gamma_{k}^{k}\right) & \geq \sum_{i=0}^{l-1} \alpha_{G}\left(\gamma_{k}^{k}\left(t_{i+1}\right), \gamma_{k}^{k}\left(t_{i}\right)\right) \geq \frac{1}{1+\epsilon} \sum_{i=0}^{l-1} \alpha_{G}\left(\gamma_{\infty}\left(t_{i+1}\right), \gamma_{\infty}\left(t_{i}\right)\right) \\
& >\frac{\alpha_{G}\left(\gamma_{\infty}\right)-\delta / 2}{1+\epsilon}=\frac{m+\delta / 2}{1+\delta /(4 m+\delta)}=m+\delta / 4
\end{aligned}
$$

This contradicts the fact that $\gamma_{k}^{k}$ is a sequence with $\alpha_{G}\left(\gamma_{k}^{k}\right) \rightarrow m$ as $k \rightarrow \infty$, which means that the assumption $\delta>0$ was false and so $\alpha_{G}\left(\gamma_{\infty}\right)=m$, which was to be shown. 


\section{Quasi-isotropy.}

Recall that the concept of quasi-isotropy was defined in Section 2.3. In this section we study bilipschitz equivalences of the Apollonian inner metric, the quasihyperbolic metric and the $j_{G}$ metric.

We denote $d \approx d^{\prime}$ if there exists a constant $C>0$ such that $\frac{1}{C} d(x, y) \leq$ $d^{\prime}(x, y) \leq C d(x, y)$ for all $x, y$ in the domain of definition of the metrics. We denote $d \lesssim d^{\prime}$ if there exists a constant $C>0$ such that $d(x, y) \leq C d^{\prime}(x, y)$ for all $x, y$ in the domain of definition of the metrics.

Proposition 5.1. For $G \varsubsetneqq \mathbb{R}^{n}$ we have $\tilde{\alpha}_{G} \approx k_{G}$ if and only if $G$ is quasiisotropic.

Proof. If $G$ is quasi-isotropic, then $\tilde{\alpha}_{G} \approx k_{G}$ by [8, Corollary 5.4].

Suppose then that $\tilde{\alpha}_{G} \approx k_{G}$. This means that there exists a constant $K$ such that $\tilde{\alpha}_{G}(x, y) \geq k_{G}(x, y) / K$ for every $x, y \in G$. Fix $x \in G$ and $e \in S^{n-1}$. We have $\tilde{\alpha}_{G}([x, x+r e]) \geq k_{G}([x, x+r e]) / K$ for every $r<\delta(x)$. Since these two metrics are integrals over continuous kernels we see by considering $r \rightarrow 0$ that $\bar{\alpha}_{G}(x ; e) \geq 1 /(K \delta(x))$. This implies that $\bar{\alpha}_{G}(x ; e) \delta(x) \geq 1 / K$ for all $x \in G, e \in S^{n-1}$ and so it follows from Lemma 2.3 that $G$ is quasi-isotropic.

Lemma 5.2. Let $x \in G$ and $y \in B^{n}(x, \delta(x))$. Then $\log \{2\}|x-y| / \delta(x) \leq$ $j_{G}(x, y)$.

Proof. It suffices to show that

$$
\log \{2\}|x-y| / \delta(x) \leq \log \{1+|x-y| / \delta(x)\}
$$

since the right hand side is less than or equal to $j_{G}(x, y)$. Since the function $z \mapsto \log \{1+z\}-z \log 2$ is increasing for $z \leq(\log 2)^{-1}-1$ and then decreasing it suffices to check this inequality for the maximal $|x-y| / \delta(x)$, i.e 1 , in which case the inequality reduces to $\log 2 \leq \log 2$.

Proposition 5.3. If $G \varsubsetneqq \mathbb{R}^{n}$ is not quasi-isotropic then $j_{G} \not \tilde{\alpha}_{G}$.

Proof. For every $K>2$ there exists a point $x \in G$ and a direction $e \in$ $S^{n-1}$ such that $\bar{\alpha}_{G}(x ; e) \delta(x)<1 / K$, since otherwise $G$ would be quasiisotropic, by Lemma 2.3. By continuity there exists a $0<r<\delta(x)$ such 
that $\bar{\alpha}_{G}(y ; e) \delta(x)<2 / K$ for all $y \in B^{n}(x, r)$. Let $z, w \in B^{n}(x, r)$ be such that $z-x=x-w=r e / 2$. Then

$\tilde{\alpha}_{G}(z, w) \leq \alpha_{G}([z, w])<\frac{2|z-w|}{K \delta(x)} \leq \frac{2}{K \log 2} \frac{\delta(z)}{\delta(x)} j_{G}(z, w) \leq \frac{3}{K \log 2} j_{G}(z, w)$.

Here the second to last inequality follows from Lemma 5.2. Since $K>2$ can be arbitrarily large, we see that $j_{G} \lesssim \tilde{\alpha}_{G}$ does not hold, as claimed.

By Lemma 2.2, the following corollary is equivalent to Theorem 1.6.

Corollary 5.4. For $G \varsubsetneqq \mathbb{R}^{n}$ the following conditions are equivalent:

1. $G$ is quasi-isotropic;

2. $k_{G} \approx \tilde{\alpha}_{G}$

3. $j_{G} \lesssim \tilde{\alpha}_{G}$.

Proof. That items 1 and 2 are equivalent was show in Lemma 5.1. In Lemma 5.3 we showed that 3 implies 1 . It thus suffices to prove that 2 implies 3. But if $\frac{1}{C} k_{G} \leq \tilde{\alpha}_{G}$, then it is clear that $j_{G} \leq C \tilde{\alpha}_{G}$ since $j_{G} \leq 2 k_{G}$ always holds.

\section{References.}

[1] Dan Barbilian, Einordnung von Lobatschewsky's Maßbestimmung in gewisse allgemeine Metrik der Jordanschen Bereiche, Casopsis Mathematiky a Fysiky 64 (1934-35), 182-183.

[2] Alan F. Beardon, Geometry of Discrete Groups, Graduate text in mathematics 91, Springer-Verlag, New York, 1995, MR 97d:22011.

[3] The Apollonian metric of a domain in $\mathbb{R}^{n}$, Quasiconformal mappings and analysis (Ann Arbor, MI, 1995) (Peter Duren, Juha Heinonen, Brad Osgood and Bruce Palka, eds.), Springer-Verlag, New York, 1998, pp. 91-108, MR 99k:30075.

[4] Wladimir-George Boskoff, Hyperbolic geometry and Barbilian spaces, Istituto per la Ricerca di Base, Series of Monographs in Advanced Mathematics, Hardronic Press, Inc, Palm Harbor, FL, 1996, MR 97e:51017. 
[5] Fredrick W. Gehring and Kari Hag, The Apollonian metric and quasiconformal mappings, In the tradition of Ahlfors and Bers (Stony Brook, NY, 1998) (Irwin Kra and Bernard Maskit, eds.), Contemp. Math. 256, Amer. Math. Soc., Providence, RI, 2000, pp. 143-163, MR 2001c:30043.

[6] Fredrick W. Gehring and Brad G. Osgood, Uniform domains and the quasihyperbolic metric, J. Anal. Math. 36 (1979), 50-74, MR 81k:30023.

[7] Fredrick W. Gehring and Bruce P. Palka, Quasiconformally homogeneous domains, J. Anal. Math. 30 (1976), 172-199, MR 55\#10676.

[8] Peter A. Hästö, The Apollonian metric: uniformity and quasiconvexity, Ann. Acad. Sci. Fenn. Math. 28 (2003) 385-414, MR 1996444.

[9] The Apollonian metric: limits of the approximation and bilipschitz properties, Abstr. Appl. Anal. 2003 (2003), no. 20, 1141-1158.

$[10] \longrightarrow$ The Apollonian metric: quasi-isotropy and Seittenranta's metric, Comput. Methods Funct. Theory, to appear.

[11] The Apollonian metric: geometry of approximation and bilipschitz domains, preprint, http://www.helsinki.fi/ hasto/pp/.

[12] Peter Hästö and Henri Lindén, Isometries of the half-apollonian metric, Complex Var. Theory Appl., to appear.

[13] Zair Ibragimov, On the Apollonian metric of domains in $\overline{\mathbb{R}^{n}}$, Complex Var. Theory Appl. 48 (2003) 837-855, MR 2014392.

[14] Conformality of the Apollonian metric, Comput. Methods Funct. Theory 3 (2003) 397-411.

[15] Paul J. Kelly, Barbilian geometry and the Poincaré model, Amer. Math. Monthly 61 (1954), 311-319, MR 15,819a.

[16] A. G. Rhodes, An upper bound for the hyperbolic metric of a convex domain, Bull. London Math. Soc. 29 (1997), 592-594, MR 98e:30005.

[17] Pasi Seittenranta, Möbius-invariant metrics, Math. Proc. Cambridge Philos. Soc. 125 (1999), 511-533, MR 2000g:30017.

[18] Matti Vuorinen, Conformal invariants and quasiregular mappings, $J$. Anal. Math. 45 (1985), 69-115, MR 87k:30034. 
[19] Conformal Geometry and Quasiregular Mappings, Lecture Notes in Mathematics 1319, Springer-Verlag, Berlin-Heidelberg-New York, 1988, MR 89k:30021.

Department of Mathematics and Statistics

P.O. Box 68, 00014 University of Helsinki, Finland

peter.hasto@helsinki.fi

www.helsinki.fi/ hasto/

Received June 5, 2002. 